\title{
Internet de las cosas: Futuro y desafío para la epidemiología y la salud pública
}

\author{
Internet of things: The future and challenges for epidemiology and public health
}

Rodolfo Rodríguez-Gómez1* orcid.org/0000-0001-5873-4312

1 Freelance, Asesor Metodológico Investigación en Salud. Bogotá, Colombia

\section{Resumen}

Introducción: Internet vive una de las más grandes revoluciones de la historia denominada Internet de las cosas. En ella, tanto la epidemiología como la salud pública tienen gran potencial, ya que el nuevo mundo hiperconectado representará espacios de reinvención e innovación nunca antes imaginados en diversos aspectos del campo de la salud. Objetivo: Reflexionar sobre las posibilidades para la epidemiología y la salud pública ante el escenario de Internet de las cosas. Materiales y métodos: Revisión documental que incluyó textos físicos y bases de datos electrónicas. Resultados: Internet de las cosas representa para la epidemiología y la salud pública, una dimensión llena de oportunidades debido a las fuentes de datos masivos y a las tecnologías de la cuarta revolución industrial, aunque también nuevos desafíos, principalmente en cuanto a seguridad y privacidad de la información. Conclusiones: Ante la era de Internet de las cosas, para la salud en general y particularmente para la epidemiología y la salud pública, se abre la posibilidad múltiples fuentes de datos, muchas en tiempo real. Esto permitirá optimizar la perspectiva y la comprensión de numerosos eventos en salud, y con ello, lograr una atención en salud más proactiva y predictiva.

Palabras clave: Internet; tecnología; información; epidemiología; salud pública. (Fuente: DeCS, Bireme).

\begin{abstract}
Introduction: Internet is living one of the greatest revolutions in history called Internet of things. Both epidemiology and public health have great potential in it since the new hyperconnected world will represent spaces for the reinvention and innovation never before imagined in various aspects of the health field. Objective: To reflect on the possibilities for epidemiology and public health in the scenario of the Internet of things. Materials and methods: Documentary review that included physical texts and electronic databases. Results: Internet of things represents for epidemiology and public health, a dimension full of opportunities due to the massive data sources and technologies of the fourth industrial revolution, but also new challenges, mainly in terms of security and privacy of information. Conclusions: The age of Internet of things has opened up the possibility of multiple data sources, many in real time, for epidemiology and public health. This will facilitate the optimization of the perspective and understanding of multiple health events and the achievement of more proactive and predictive health care.
\end{abstract}

Key words: Internet; technology; information; epidemiology; public health. (Source: DeCS, Bireme).

\footnotetext{
*Autor de correspondencia

Rodolfo Rodríguez Gómez

e-mail: fitopolux@hotmail.com
} 


\section{Introducción}

Una nueva revolución transforma el mundo digital en la actualidad. Esta innovación es una evolución de Internet(1), aquella tecnología que ha modificado, como pocos sucesos en la historia de la humanidad, la vida del ser humano y la sociedad. Internet, desde algunos años atrás, explora terrenos vanguardistas donde los objetos estarán hiperconectados y serán, en cierta medida, conscientes del entorno mediante la integración de dispositivos de rastreo y sensores cableados e inalámbricos(1). Hoy en día es difícil imaginar un escenario donde la sociedad se prive de Internet, ya que la conectividad y sus prácticas significantes impactan en áreas sociales, económicas y culturales. Sin embargo, el mundo digital gravitará en un área de integración entre la gente y los aparatos, donde se conectarán el mundo físico con los entornos virtuales creados por el hombre(2), y esta será la dimensión de la hiperconectividad. Para el área de la salud, la era digital representa nuevos escenarios que modifican la práctica asistencial, la educación y la investigación. Desde historias clínicas digitales, bases de datos científicas, software de análisis estadístico, plataformas Moodle, robótica quirúrgica, hasta la telemedicina(3), todo tiene que ver con lo digital. No obstante, la revolución denominada Internet de las cosas está llamada a generar un cambio trascendente del mundo como se conoce hoy en día, dado que, en el desarrollo de todo su potencial, el área de la salud dispondrá, como nunca antes, de datos masivos en forma ubicua y en tiempo real.

\section{Materiales y métodos}

El presente artículo se basó en una búsqueda documental, principalmente libros físicos $\mathrm{y}$ documentos en bases de datos electrónicas con las palabras clave en español: internet de las cosas, ciberespacio, internet, conectividad, salud móvil, salud, epidemiología y salud pública. En inglés; internet, internet of things, connectivity, wearables, wearables, eHealth, health, epidemiology, public health. Las principales bases de datos consultadas fueron: PubMed, Science Direct, Ovid, Google Scholar, Google Académico y Google Books. También se consultaron sitios web corporativos e institucionales (Cisco, Naciones Unidas, European Commission, Banco Interamericano de Desarrollo, Organización Mundial de la Salud, entre otros). Aunque no se aplicó restricción idiomática, la búsqueda se concentró en documentos en inglés y español. No se estableció límite en cuanto a fechas, sin embargo, dadas las características del tema, se prestó especial atención en documentos de reciente publicación. Las búsquedas se realizaron entre abril de 2017 y abril de 2019, recuperando cerca de 70 documentos considerados potenciales de los cuales 45 se incluyeron como referencias del artículo. La información recopilada fue revisada y los contenidos analizados con el fin de extraer conceptos y categorías para estructurar el documento.

\section{Resultados}

\section{¿Qué es Internet de las Cosas?}

El término 'Internet de las cosas' se acuñó a finales de la década de los noventa del siglo XX(4). Se atribuye al británico Kevin Ashton quien durante su trabajo en Procter \& Gamble, planteó que los objetos de la vida cotidiana deberían ser capaces de introducir información a la web sin ayuda de las personas(5). Una década atrás, el científico Mark Weiser había dado los primeros pasos en la integración de la informática con el entorno de los individuos en lo que se conoció como 'computación ubicua'(6). Dicha innovación tecnológica, hace referencia a sistemas informáticos que están presentes en todo: la ropa, las casas, los vehículos, los lugares de trabajo, los accesorios de uso cotidiano e incluso en las partes del cuerpo humano(6). Este concepto denominado como Internet de las cosas, abrió una nueva senda e inspiró a otros como, por ejemplo, al físico Neil Gershenfeld, quien en 2001 planteó un escenario donde las cosas reconocieran las necesidades de las personas(7).

Internet de las cosas representa entonces una nueva dimensión digital donde gran cantidad de objetos adquieren mayor capacidad de cómputo y toman, en cierta medida, consciencia del entorno mejorando su poder de procesamiento y su independencia energética( ${ }^{(8)}$. Es un mundo donde se empodera a los ordenadores para recopilar datos de las cosas y el ambiente que los rodean gracias a sensores y a la Radio Frequency Identification System (RFID), tecnología automática que permite a las máquinas o computadores identificar y entender el entorno a través de ondas de radio sin ayuda de los seres humanos y esto, en tiempo real( ${ }^{9}$. Vale la pena recalcar que Internet de las cosas es mucho más que conectar cosas, de hecho, es un concepto análogo a lo que representa Internet, que es mucho más que unos ordenadores conectados entre sí. Es, en otras 
palabras, interacción digital llevada a otro nivel, uno donde la relación meramente instrumental con la tecnología pasa a convertirse en una sinergia que hace al mundo mucho más inteligente.

\section{Internet de las cosas, wearables y salud}

En la actualidad se calcula que todo individuo está rodeado por cerca de 1000 a 5000 objetos( $^{(10)}$, donde menos del 1\% de estos se encuentran conectados a Internet. Según Cisco, uno de los mayores fabricantes de dispositivos de red en el mundo, para el año 2020 cerca de 50.000 millones de objetos estarán conectados a Internet(11). Esto quiere decir que en un futuro cercano más cosas que seres humanos estarán conectados a Internet, un fenómeno comparable con las suscripciones a telefonía celular, pues décadas atrás, la telefonía móvil era algo utópico, pero para finales de 2015, en el mundo existían cerca de 7.000 millones de suscripciones de dispositivos móviles(12). De esta manera, en el contexto de la revolución tecnológica que plantea Internet de las cosas, la nueva realidad para la sociedad será otra y el área de la salud se enfrentará a un escenario disruptivo dado que se tendrá acceso a datos masivos, tanto individuales como medioambientales.

Es claro que algunas tecnologías relacionadas con Internet de las cosas ya están en marcha, sin embargo, muchas de ellas se encuentran en fases incipientes y su completo desarrollo revierte un enorme potencial. Un ejemplo de estas tecnologías es la mHealth (mobile health), definida como 'la práctica médica y de salud pública apoyada por teléfonos celulares, dispositivos de monitorización de pacientes, tabletas, asistentes personales digitales y otros dispositivos inalámbricos'(13). La acelerada disponibilidad y expansión de teléfonos móviles en países en desarrollo ha creado múltiples oportunidades para utilizar estas tecnologías con el fin de apoyar los esfuerzos en salud(14). En países en vías de desarrollo, son múltiples los usos de la mHealth en epidemiología y salud pública como en recolección de datos clínicos y comunitarios, monitoreo de signos vitales en tiempo real, envío de mensajes de texto para solicitud de ambulancias en sitios remotos, entrega de información en salud para practicantes, investigadores y pacientes, aplicaciones para promover concientización ante enfermedades, provisión de cuidado en salud (telemedicina), soporte de diagnóstico y tratamiento, manejo de enfermedades transmisibles, educación a los pacientes, advertencias de desastres y seguimiento de brotes epidémicos(15).

Gracias a los avances digitales y de conectividad, los wearables iconos de Internet de las cosas, son todos aquellos dispositivos electrónicos o computadores que se incorporan a la ropa y a todo tipo de accesorios, que pueden ser usados por una persona y monitorear continuamente la actividad individual sin interrupción(16). Ejemplos de estos dispositivos incluyen gafas, lentes de contacto, ropa, balacas, anillos, brazaletes e incluso aretes. Así entonces, su potencial en el área de la salud es tan diverso que puede ir desde detectar arritmias hasta medir funciones corporales, parámetros de actividad física, niveles de glucosa y cualquier otro marcador biométrico. Además, tienen la ventaja de reducir costos en salud y son considerados herramientas confiables como sistema de monitoreo en salud a largo plazo(17). Así, en el contexto de esta tecnología, ya se utiliza el término Internet of Wearables Things, que combina el uso de Internet de las cosas y los wearables, que gracias a los avances en miniaturización del hardware, integración y conectividad, han permitido la irrupción de Internet de las cosas, que conecta dispositivos como robots y sensores a Internet ${ }^{(18) .}$

En la industria de la salud una de las áreas más prometedoras es el de las aplicaciones de Internet de las cosas. Esta tecnología es una realidad en el área de la salud y de hecho, ya se ha acuñado el término Internet of Medical Things, lo cual significa que puede ser usada, en cierta medida, para el monitoreo de enfermedades a través de la captura de bioseñales(19). En la actualidad, la tecnología RFID ya se utiliza en ensayos clínicos para monitorizar a los participantes siendo de gran utilidad para la investigación clínica y la epidemiología por proveer en tiempo real, datos de trazabilidad, identificación, comunicación, temperatura y ubicación para personas y recursos ${ }^{(20)}$. Todo ello es el efecto de la informática ubicua trasladada al área de la salud en esta era, la de la telemetría, donde se crea información a partir de las actividades cotidianas de las personas(21). Su uso permitirá el acceso a datos masivos sobre la salud poblacional, muchos de ellos en tiempo real, y aunque su uso individual puede ser de enorme beneficio para la medicina clínica, a gran escala representa una revolución para la salud global en campos como la detección temprana, la prevención de la enfermedad y la promoción de la 
salud, los sistemas de monitorización, los sistemas de emergencias, la telemedicina y la investigación biomédica.

\section{Tecnología al servicio de la salud, la epidemiología y la salud pública}

Ante el futuro que se avecina con Internet de las cosas cabe preguntar $¿$ En que se relaciona esto con la epidemiología o la salud pública? La respuesta es, como ya se ha mencionado, en todo, pues, precisamente, la materia prima de la epidemiología y la salud pública son los datos, y en el contexto de Internet de las cosas, con la computación ubicua, los wearables, la telemedicina, el cloud computing, la mHealth y el big data, se contará con datos relacionados con infinidad de eventos en salud humanidad. Además, es claro que en el planeta todo está intimamente interrelacionado, y en este sistema de gran complejidad, buen número de problemas epidemiológicos y de salud pública están asociados con cosas que rodean a los individuos y sus ecosistemas, ya sea por los objetos per se, o por el uso que se hace de ellos. En este orden de ideas, si cierto elemento, potencialmente tóxico o peligroso, está involucrado con actividades de la vida cotidiana, de una u otra forma lo estará con la salud poblacional, por tanto, tendrá que ver con la salud pública.

En las grandes ciudades, por ejemplo, cada vez emergen aspectos más críticos para la salud pública. Para el año 1950 existían sólo dos megaciudades: la zona metropolitana de New York y Tokio(22). Pero para la década de los noventa se sumaron ocho más engrosaron la lista: Buenos Aires, Calcuta, Ciudad de México, Los Ángeles, Mumbai, Osaka-Kobe, San Pablo y Seúl(23), sin embargo, para 2025 se proyecta que existirá un total de 37 megalópolis, de las cuales 30 estarán en países en desarrollo(23). Estos centros urbanos de alta densidad demográfica, enfrentan infinidad de desafíos, los cuales son cada vez más complejos e impactan en mayor o menor grado en la salud poblacional y la salud global. Dentro de ellos se incluyen fenómenos como la escasez y calidad del agua, las enfermedades transmisibles $y$ no transmisibles, la falta de planificación urbanística, la contaminación atmosférica y de fuentes hídricas, los efectos del cambio climático, el aumento de desechos y productos tóxicos, el desperdicio de alimentos, el déficit de la atención sanitaria, los problemas de salud mental y los accidentes de tráfico, entre otros.
Un gran problema de salud pública en las megaciudades son los accidentes de tránsito. Actualmente, cerca de 1,3 millones de personas mueren cada año como consecuencia de este tipo de eventos(24), los cuales representan la principal causa de muerte de niños y adultos jóvenes entre los 5 y 29 años(25). Pero en el contexto de Internet de las cosas, en caso de accidente, la tecnología eCall, al estar conectada al automóvil, contactará de manera automática con el número de emergencias más cercano para activar la asistencia médica a los pasajeros involucrados(26). Otro avance trascendental será el vehículo autónomo, algo que décadas atrás se enmarcaba dentro de la ciencia ficción, pero ya es una realidad y en un futuro cercano, quizás la gran mayoría o todos los vehículos prescindirán del conductor. De esta manera, dado que la gran mayoría de los accidentes de tráfico son ocasionados por errores humanos, los vehículos robóticos prometen generar un impacto dramático en el fenómeno de este tipo de accidentes.

Algo relevante que permitirá Internet de las cosas será trascender la gestión intermitente y a corto plazo de algunos problemas de salud. En este contexto, será de gran valor contar con una recopilación longitudinal tanto de datos personalizados, como de datos medioambientales, algo útil para el seguimiento de los eventos de interés epidemiológico (27). Por otro lado, el objetivo con los datos personalizados es lograr intervenciones más proactivas, muchas de las cuales estarán encaminadas a cambios en el estilo de vida, por consiguiente, el futuro de la salud individual y la salud pública dependerá, en buena medida, de cuánto se aprenda de estas estrategias que se convertirán en mejoras basadas en la evidencia(27). En este punto, la denominada Augmented Personalized Healthcare (APH) pretende potenciar el cuidado de la salud al sacar provecho de los datos personalizados obtenidos mediante Internet de las cosas con el uso de sensores, wearables, aplicaciones móviles, Electronic Medical Records (EMRs) y social media, utilizando técnicas de inteligencia artificial para mejorar la salud y el bienestar poblacional(28).

Gracias a la Inteligencia Artificial, las máquinas tienen la capacidad de captar información del entorno, resolver problemas y adaptarse al contexto, es decir, son inteligentes ${ }^{(29)}$. En la actualidad, muchas cosas se catalogan así: teléfonos inteligentes, universidades inteligentes, hogares inteligentes, 
escuela inteligente, estadio inteligente, fábrica inteligente, tutor inteligente, señalización inteligente, iluminación inteligente, autos inteligentes, semaforización inteligente, y ciudad inteligente(29). Estos sistemas constituyen un nuevo paradigma y ante el presente-futuro que representa Internet de las cosas, millones de dispositivos estarán conectados a Internet y enviarán todo tipo de información sobre infinidad de eventos como parámetros biométricos de gran cantidad de personas, condiciones atmosféricas, alertas sísmicas, niveles de contaminación de fuentes hídricas, exposición a radiación ultravioleta, niveles de material particulado, calidad del aire en las casas, monitoreo de biodiversidad, caducidad de los alimentos, accidentes viales, etc. Todo ello tiene que ver con la salud pública, y ante ese panorama, se podría hablar entonces de una salud pública inteligente, un contexto digital vanguardista donde esta disciplina sea proactiva en los procesos de innovación en salud $\mathrm{y}$, principalmente, en la comprensión, la prevención y la anticipación de los eventos en salud.

La inteligencia artificial ha logrado increíbles avances en salud, pero un área de gran impacto es la telemedicina, la cual está dando frutos en el contexto de la salud pública. Las innovaciones en este ámbito brindan soluciones reales al problema de la asistencia remota en salud y de hecho, está transformando el desarrollo de nuevos modelos de atención con el uso de tele-evaluación, telediagnóstico, tele-interacciones y telemonitoreo(30). Esto es algo disruptivo para el campo de la salud, dado que poblaciones de bajos recursos y ubicación geográfica remota pueden tener acceso a determinadas acciones en salud, que junto al desarrollo de drones para casos particulares, generaría grandes beneficios especialmente en países en vías de desarrollo donde la distancia entre zona urbana y rural, afecta el acceso a los servicios de salud. Las innovaciones en telemedicina pueden reducir dichas distancias al potenciar el acceso a servicios médicos para las comunidades desatentidas, aunque para esto se requiere de una fuerte colaboración intersectorial además de un adecuado manejo de los recursos tecnológicos para lograr una innovación sostenible en telesalud rural(31).

En cáncer, un importante problema de salud pública a nivel mundial, Internet de las cosas y la inteligencia artificial están haciendo aportes significativos. De hecho, estas tecnologías se están convirtiendo en los mejores aliados en la lucha contra esta enfermedad que se ha convertido en una epidemia, ya que anualmente genera millones de nuevos casos alrededor del mundo. Hoy en día, novedosos sistemas de detección de cáncer que usan deep learning se están utilizando en las radiografías analizadas y para la predicción del crecimiento de neoplasias. CancerLinQ, por ejemplo, una iniciativa de la American Society of Clinical Oncology, está diseñada para el análisis de datos de registros electrónicos en salud, ensayos clínicos y guías de práctica clínica, todo esto aplicando metodologías de big data para direccionar la investigación del cáncer(32). Algo trascendental para la investigación y la epidemiología como disciplina esencial de la salud pública, es que este tipo de tecnologías no sólo permiten optimizar la recolección y el análisis de datos masivos, sino encontrar asociaciones entre variables para optimizar la comprensión del cáncer.

\section{Desafíos de Internet de las cosas en salud}

En un mundo hiperconectado, una de las principales amenazas es la seguridad de la información. En salud, esto es particularmente importante porque puede contener datos sensibles y confidenciales que al transmitir a través de conexiones inalámbricas es probable que fácilmente sea interceptada, modificada o incluso destruida(33). Dado que el almacenamiento de los datos en salud y de tecnologías como mHealth recae en la computación en la nube, dicho servicio puede ser víctima de ataques cibernéticos, pues se ha evidenciado fallas de seguridad(34). Así, una de las principales características de los dispositivos que recopilan información en salud, puede convertirse en su principal desventaja, ya que tecnologías como mHealth crearán vulnerabilidades donde antes no existían(34), en consecuencia, la sociedad se verá enfrentada a un nuevo nivel de riesgo en cuanto a la seguridad de su información.

En el contexto de datos en salud surge la pregunta en relación a quién es el propietario de los mismos. Este no es un tema banal, ya que la información puede ser de gran valor tanto para el área clínica, la investigación, e incluso el área comercial, motivo por el cual se requiere debatir sobre quién tiene la legítima potestad para autorizar el uso de los datos e incluso el lucro con su uso(34). Dado que tras estas innovaciones tecnológicas existe una enorme 
cantidad de elementos de propiedad intelectual, especialmente en lo que concierne al desarrollo e implementación, por ende en los temas relacionados con la propiedad de los datos no son menores y requieren amplios debates y mecanismos regulatorios claros y específicos por las organizaciones correspondientes. Uno de los aspectos claves es la transparencia de la tecnología y cómo esa tecnología maneja los datos, así como el proveer opciones para el usuario basándose en el concepto de Ethical Design implementado gracias a un marco regulatorio basado en políticas(35). Recolectar datos de salud a través de dispositivos también genera desafíos en cuanto a cómo, cuándo y con quién compartir los datos, además de qué tipo de información compartir, con qué especificidad y para qué va a ser utilizada(36). Dado que buena parte de la información recolectada por sistemas de Internet de las cosas puede ser personal e íntima, la confidencialidad y el anonimato es muy relevante, por tal motivo, los datos deben permanecer sujetos a políticas de acceso cuando se usen para investigación o propósitos de salud pública donde la identificación no es necesaria(36).

La privacidad de los datos es un aspecto ético que genera gran preocupación. En salud, los dispositivos podrán recopilar información sobre todo tipo de variables, muchas de ellas ni siquiera pensadas años atrás y esto representará nuevos desafíos en privacidad. En este tema, la Organización Mundial de la Salud (OMS) ha trabajado en aspectos claves del estado actual del marco regulatorio alrededor del mundo(37), sin embargo, en la mayoría de países los controles al respecto aún están en fases tempranas. En Europa, por ejemplo, la European Data Protection Directive establece derechos básicos de privacidad, pero existen variaciones sustanciales de implementación de esos derechos entre los países miembros(37). Con el creciente número de objetos conectados a internet recolectando datos en salud, los problemas de seguridad y privacidad serán cada vez más desafiantes. Hoy en día, esta es una preocupación primordial para la industria del cuidado en salud debido a consecuencias catastróficas como tratamiento inadecuado de la información y pérdidas financieras(38). Por consiguiente, al pensar en recolección de datos para el área clínica y la salud pública, existe una urgente necesidad de identificar y evaluar los riesgos y con ello mejorar la toma de decisiones al adoptar y diseñar un Internet of Medical Things más seguro y confiable(38).

Algo paradójico recae en que las nuevas tecnologías como Internet de las cosas, las mismas que están revolucionando las fuentes de información para la atención clínica y la salud pública, pueden impactar negativamente en la salud medioambiental y por consiguiente, en la salud poblacional. Estudios como el del Centre for Energy-Efficient Telecommunications (CEET), estiman que la industria de telecomunicaciones produce más de 830 millones de toneladas de dióxido de carbono al año, y según los pronósticos, esas cifras se duplicarán para 2020(39). El impacto medioambiental proviene de la energía necesaria para hacer funcionar la infraestructura, donde los mayores consumidores de electricidad son los centros de datos. Según Greenpeace, gran cantidad de la energía que requieren estos centros proviene de instalaciones de carbón, lo que supone la emisión de miles de toneladas de dióxido de carbono a la atmósfera(40). Esto plantea grandes retos para reducir la huella ecológica, ya que no solo son los centros de datos los que contaminan, sino también las redes de acceso y los equipos que se usan para acceder a Internet.

\section{Discusión}

Lo que se avecina con Internet de las cosas y la cuarta revolución industrial, especialmente en relación a la salud, la epidemiología y la salud pública, será un escenario bastante disruptivo, pero lleno de oportunidades para lograr una medicina más eficiente, asequible y precisa(41). En una época globalizada como la actual, lo concerniente a la salud pública tiene que ver con todo, o casi todo, y es difícil pensar en un sector o área que no tenga que ver con ella; está en el agua que se bebe, en la comida que se ingiere, en el aire que se respira, en el medioambiente, en los productos que se usan y desechan, en el sistema de atención sanitaria, en los sistemas de transporte(42), etc. Asimismo, en un mundo colmado de sensores, algoritmos e inteligencia artificial, las fuentes de datos para la epidemiología y la salud pública serán casi que omnipresentes. En la actualidad, uno de los elementos críticos para la toma de decisiones en salud es la debilidad en la información(43), ya sea por datos que no existen, datos que no se recopilan de manera sistemática o errores e imprecisiones en los datos, principalmente, por errores humanos. Con 
Internet de las cosas esto cambiará de manera trascendental, ya que se recopilará información de infinidad de variables a una escala y con una precisión inimaginable, y esto permitirá optimizar la perspectiva y la comprensión sobre gran cantidad de eventos en salud.

El futuro con Internet de las cosas apunta a una medicina más personalizada y predictiva(44). Ofrecerá oportunidades sin precedentes para lograr dejar de apagar fuegos y apuntar, realmente, a una atención en salud más proactiva y preventiva, donde los algoritmos y el aprendizaje automático de las máquinas llevarán a otro nivel la investigación en salud. Por supuesto que existen retos y desafíos como lo relacionado con la seguridad de la información, la privacidad de los datos(45), y lograr ser energéticamente eficientes al desarrollar fuentes de energía renovables que permitan a Internet de las cosas un desarrollo sostenible y que no impacte de forma negativa, precisamente, en la salud pública. No obstante, con los datos masivos el problema en el futuro cercano no será entonces el cómo y dónde obtener información, sino por el contrario, recaerá en qué hacer con tanta información. Será un contexto donde ya no se investigará lo que se pueda, sino lo que se quiera, lo cual representará infinidad de ventanas de oportunidad no solo para la epidemiología y la salud pública, sino para toda el área de la salud.

\section{Conclusiones}

El nuevo escenario que se vislumbra con Internet de las cosas recalca que el mundo avanza a mayor velocidad de lo que se cree. Hoy en día, las dinámicas son mucho más intensas y complejas, de hecho, en la historia de la humanidad, nunca antes el presente y el futuro habían estado tan cerca uno del otro, casi que sobrepuestos en la línea del tiempo. Por ello, especialmente en salud, la tarea al imaginar el futuro es hablar del presente y antes que nada, entender la nueva dimensión digital que ya es una realidad. Es claro que las reflexiones en este tema no solo apuntan a las grandes oportunidades con los datos masivos, sino también a los desafíos en torno a la seguridad y la privacidad, temas complejos que trascienden el campo de la salud y requieren abordajes interdisciplinarios. Internet de las cosas, representa para el área de la salud, la epidemiología y la salud pública, posibilidades innovadoras para entender muchos fenómenos en salud gracias a los algoritmos y la inteligencia artificial, sin embargo, uno de los principales retos será lograr pasar de acumular datos a transformarlos en investigación y acción en salud en tiempo real. Así, Internet de las cosas puede ser un espacio digital donde el área de la salud gravite en la misma orbita con los avances tecnológicos, pero al mismo tiempo, de la mano de políticas públicas y mecanismos regulatorios apropiados, se beneficie a la sociedad optimizando la salud individual y la salud pública.

\section{Conflicto de intereses}

Los autores afirman que no existen conflictos de interés.

\section{Referencias}

1. Yager RR, Espada JP. New advances in the Internet of Things. USA: Springer Science \& Business Media; 2017.

2. Buyya R, Vahid A. Internet of Things: principles and paradigms. Cambridge (USA): Elsevier; 2016.

3. Carrión P, Ródenas J, Rieta J, Sánchez C. Telemedicina: ingeniería biomédica. España: Ediciones de la Universidad de Castilla La Mancha; 2009.

4. Saleh I, Ammi M, Szoniecky S. Challenges of the Internet of Things: Technique, Use, Ethics. Vol. 7. USA: John Wiley \& Sons, Inc; 2018.

5. Zanoni L. Futuro inteligente. Objetos, casas, datos y ciudades: el mundo conectado. Buenos Aires (Argentina): Recursos Editoriales; 2014.

6. Ekman U, Bolter JD, Diaz L, Sondergaard M, Engberg M. Ubiquitous Computing, complexity and culture. New York: Routledge; 2016.

7. Gershenfeld N. Cuando las cosas empiecen a pensar. Barcelona (España): Ediciones Granica; 2000.

8. Evans D. The Internet of Everything. How More Relevant and Valuable Connections Will Change the World [Internet]. USA: Cisco Internet Business Solutions Group (IBSG); 2012 [citado 28 de marzo de 2019]. Disponible en: https://www.cisco.com/c/dam/global/en_my/assets/cisc oinnovate/pdfs/IoE.pdf

9. Agbaeze A, Abdul SK, Yen C. RFID in the Internet of Things. En: Internet of Things A to Z: Technologies and Applications. New Jersey: John Wiley \& Sons, Inc; 2018.

10. Hua H. Mobile Marketing Management: Case Studies from Successful Practices. New York (USA): Routledge; 2019.

11. Evans D. Internet de las cosas. Cómo la próxima evolución de Internet lo cambia todo [Internet]. Cisco Internet Business Solutions Group (IBSG); 2011 [citado 27 de marzo de 2019]. Disponible en: https://www.cisco.com/c/dam/global/es_mx/solutions/e xecutive/assets/pdf/internet-of-things-iot-ibsg.pdf

12. International Telecommunications Union. Facts \& Figures. The World in 2015 [Internet]. Geneva: ITU; 2015 [citado 27 de marzo de 2019]. Disponible en: https://www.itu.int/en/itu-

d/statistics/documents/facts/ictfactsfigures2015.pdf

13. World Health Organization. Frequently asked questions on Global Task Force on digital health for TB and its work. Geneva: WHO; 2015. 
14. Wahl B, Cossy-Gantner A, Germann S, Schwalbe NR. Artificial intelligence (AI) and global health: how can AI contribute to health in resource-poor settings? BMJ Glob Health. 2018;3(4):e000798.

15. Davey S, Davey A. Mobile-health technology: Can it Strengthen and improve public health systems of other developing countries as per Indian strategies? A systematic review of the literature. Int $\mathrm{J}$ Med Public Health. 2014;4(1):40-5.

16. Gao W, Emaminejad S, Nyein HYY, Challa S, Chen K, Peck A, et al. Fully integrated wearable sensor arrays for multiplexed in situ perspiration analysis. Nature. 2016;529(7587):509-14.

17. Haghi M, Thurow K, Stoll R. Wearable Devices in Medical Internet of Things: Scientific Research and Commercially Available Devices. Heal Inf Res. 2017;23(1):4-15.

18. Ometov A, Bezzateev S, Kannisto J, Harju J, Andreev S, Koucheryavy Y. Facilitating the Delegation of Use for Private Devices in the Era of the Internet of Wearable Things. IEEE Internet Things J. 2017;4(4):843-54.

19. Qureshi F, Krishnan S. Wearable Hardware Design for the Internet of Medical Things (IoMT). Sens Basel. 2018;18(11):3812.

20. Paaske S, Bauer A, Moser T, Seckman C. The Benefits and Barriers to RFID Technology in Healthcare. Online J Nurs Inform OJNI [Internet]. 2017;21(2). Disponible en: http://www.himss.org/ojni

21. Tucker P. The Naked Future: What happens in a world that anticipates your every move? New York (USA): Penguin Group; 2014.

22. Franklin D, Andrews J. El mundo en 2050: todas las tendencias que cambiarán en el planeta. Barcelona (España): Grupo Planeta; 2013.

23. Banco Interamericano de Desarrollo. COP21 y el desarrollo urbano. ¿Hacia dónde debemos dirigir nuestro barco planetario?. Washington: BID; 2015.

24. Neelakantan A, Kotwal BA, Ilankumaran M. Determinants of injuries and Road Traffic Accidents amongst service personnel in a large Defence station. Med J Armed Forces India. 2017;73(3):216-21.

25. World Health Organization. Global Status Report On Road Safety 2018. Geneva: WHO; 2018.

26. Carutasu G. Further challenges of eCall service and infrastructure [Internet]. Slovenia: MIT; 2016 [citado 28 de marzo de 2019]. Disponible en: https://pdfs.semanticscholar.org/2529/ba9ead9610e7702 30ea3c14c7064b44ee310.pdf

27. Sheth A, Jaimini U, Yip HY. How Will the Internet of Things Enable Augmented Personalized Health? IEEE Intell Syst. 2018;33(1):89-97.

28. Sheth A, Jaimini U, Thirunarayan K, Banerjee T. Augmented Personalized Health: How Smart Data with IoTs and AI Is about to Change Healthcare. Proc IEEE 3rd Int'l Forum Res Technol Soc Ind RTSI. 2017;1-6. DOI: 10.1109/RTSI.2017.8065963

29. Fundación Telefónica. Máquinas inteligentes en un mundo de sensores. España: Planeta Spain; 2016.

30. Kuziemsky C, Maeder AJ, John O, Gogia SB, Basu A, Meher S, Ito M. Role of Artificial Intelligence within the Telehealth Domain. Yearb Med Inf. 2019;28(1):35-40. doi: 10.1055/s0039-1677897

31. Singh R, Mathiassen L, Stachura ME, Astapova EV. Sustainable Rural Telehealth Innovation: A Public Health Case Study. Health Serv Res. 2010;45(4):985-1004.
32. Chung $\mathrm{AE}$, Jensen RE, Basch EM. Leveraging Emerging Technologies and the "Internet of Things" to Improve the Quality of Cancer Care. J Oncol Pr. 2016;12(10):863-6.

33. Bajwa M. mHealth Security. Pak J Med Sci. 2014;30(4):9047.

34. Cvrkel T. The ethics of mHealth: Moving forward. J Dent. 2018;74:S15-20.

35. Baldini G, Botterman M, Neisse R, Tallacchini M. Ethical Design in the Internet of Things. Sci Eng Ethics. 2018;24(3):905-25.

36. Kotz D, Gunter C, Kumar S, Weiner JP. Privacy and Security in Mobile Health: A Research Agenda. Comput (Long Beach Calif). 2016;49(6):22-30.

37. World Health Organization. Legal Frameworks for eHealth: Based on the Findings of the Second Global Survey on eHealth. Global Observatory for eHealth Series [Internet]. Geneva: WHO; 2012 [citado 29 de marzo de 2019]. Disponible

en: https://apps.who.int/iris/bitstream/handle/10665/44807 /9789241503143_eng.pdf;jsessionid=591BD3100E0DFF9F 9CB82F86F55EABFC? sequence $=1$

38. Alsubaei F, Abuhussein A, Shiva S. Security and Privacy in the Internet of Medical Things: Taxonomy and Risk Assessment. In: 2017 IEEE 42nd Conference on Local Computer Networks Workshops (LCN Workshops). 2017;72:112-20.

39. Chan CA, Gygax AF, Wong E, Leckie CA, Nirmalathas A, Kilper DC. Methodologies for Assessing the Use-Phase Power Consumption and Greenhouse Gas Emissions of Telecommunications Network Services. Env Sci Technol. 2013;47(I):485-92.

40. Greenpeace International. How clean is your cloud? [Internet]. Greenpeace; 2012 [citado 27 de marzo de 2019]. Disponible en: https://www.greenpeace.org/archiveinternational/Global/international/publications/climate/2 012/iCoal/HowCleanisYourCloud.pdf

41. World Economic Forum. Health and Healthcare in the Fourth Industrial Revolution. Global Future Council on the Future of Health and Healthcare 2016-2018 [Internet]. World Economic Forum; 2019 [citado 8 de agosto de 2019]. Disponible en: http://www3.weforum.org/docs/WEF_Shaping_the_Futur e_of_Health_Council_Report.pdf

42. World Health Organization. Transporte urbano y salud. Transporte sostenible: texto de referencia para formuladores de políticas públicas de ciudades en desarrollo [Internet]. WHO, Geneva; 2011 [citado 8 de agosto de 2019]. Disponible en: https://www.who.int/hia/green_economy/giz_transport_s p.pdf?ua $=1$

43. Bernal-Acevedo O, Forero-Camacho JC. Sistemas de información en el sector salud en Colombia. Rev Gerenc Polit. 2011;10(21):85-100.

44. Dimitrov DV. Medical Internet of Things and Big Data in Healthcare. Heal Inf Res. 2016;22(3):156-63.

45. Rosner G, Kenneally E. Privacy and the Internet of Things. Emerging Frameworks for Policy and Design [Internet]. Center for Long-Term Cybersecurity; 2018 [citado 8 de agosto de 2019]. Disponible en: https://cltc.berkeley.edu/wpcontent/uploads/2018/06/CLTC_Privacy_of_the_IoT-1.pdf 